\title{
200 Jahre TU Wien: Vom Versuch, ein Jubiläum (fast) ohne Geschichte zu feiern
}

\begin{abstract}
The 200 Years Anniversary of TU Wien in 2015 happened in a difficult context: Like all Austrian Universities, TU Wien since 2004 had experienced a period of far reaching organizational reforms, challenging, among other things, the corporate identity of its members. In addition, it had to compete with two other Viennese universities (Vienna University and Vienna University of Veterinary Medicine), who also celebrated their anniversaries in 2015, for resources and public attention. The paper examines the strategies of TU Wien to gain a maximum of attention for its unique scientific competencies and at the same time strengthen internal corporate identity. Reviewing the celebrations of earlier anniversaries, this contribution shows which festive traditions were continued and which new ways of defining and presenting a corporate image of the university were developed. In addition, the strategies of TU Wien are compared with those of its two competitors in anniversary celebration, using commemorative publications and other media, to point out differences and similarities of the respective festive cultures.
\end{abstract}

\section{Einleitung}

Das 200-Jahr-Jubiläum der Technische Universität Wien (TU Wien) im Jahr 2015 fiel in eine Zeit, in der sich das österreichische Universitätssystem in einem grundlegenden Umbruch befand. Durch die Umsetzung des Universitätsgesetzes 2002 (UG 02) ab dem Jahr 2004 waren die österreichischen Hochschulen rechtlich aus dem unmittelbaren Zuständigkeitsbereich des damaligen Bundesministeriums für Wissenschaft und Forschung ausgegliedert und in eine weitgehende Autonomie als Körperschaften öffentlichen Rechts entlassen worden. Dadurch wurde eine Phase tiefgreifender Umstrukturierungen eingeleitet, die natürlich auch die TU Wien betraf.

Für sie kamen noch zwei weitere Zäsuren hinzu: Erstens hatte sich das Rektorat schon 2006 gegen eine komplette Verlegung der Universität an den Stadtrand von Wien und stattdessen für den Verbleib in der Stadt entschieden. Es folgte dabei dem Ergebnis einer umfangreichen Diskussion unter Beteiligung aller Angehörigen der TU Wien. Damit begann ein bauliches Restrukturierungsprojekt, das die räumliche Konzentration und Neugestaltung aller innerstädtischen 
Standorte der TU Wien umfassen und - so der Plan - bis zum Jubiläumsjahr 2015 abgeschlossen sein sollte, weshalb es unter dem Motto „univercity2015“ firmierte. ${ }^{1}$ Zweitens fand 2011 ein Wechsel im Rektorat der TU Wien statt: Der bisherige Rektor Peter Skalicky, Professor für Angewandte Physik, wurde nach 20-jähriger Amtszeit durch die Werkstoffwissenschaftlerin und bisherige Vizerektorin für Forschung Sabine Seidler und damit erstmals durch eine Frau abgelöst.

Die TU Wien erlebte also um 2015 in kurzer Zeit gleich mehrere „Kulturbrüche“. Für das anstehende Jubiläum bedeutete dies geradezu eine Einladung, sich über eine neuerliche Standortbestimmung Gedanken zu machen. ${ }^{2}$ Ein solcher Prozess wird, implizit oder explizit, immer auch Bezug nehmen auf bereits bestehende lokale Traditionen des Gedenkens und Feierns. Daher sollen im ersten Teil meiner Darstellung frühere Jubiläen der TU Wien betrachtet und darauf hin analysiert werden, inwieweit sie die Gestaltung der 200-Jahr-Feier von 2015 beeinflusst haben. Interessieren wird hier, ob und welche Traditionen sich in den ersten 200 Jahren ihres Bestehens herausgebildet haben, welche davon 2015 aufgenommen und welche neuen Wege gesucht wurden. ${ }^{3}$

Ein weiterer Aspekt, der die Gestaltung der Jubiläumsfeierlichkeiten beeinflussen sollte, war der Umstand, dass im Jahr 2015 auch zwei weitere Wiener Universitäten große Jubiläen begingen. Die Universität Wien blickte auf ihr 650jähriges Bestehen zurück und die Universität für Veterinärmedizin Wien feierte den 250. Jahrestag ihrer Gründung. Dieses zeitliche Zusammenfallen der drei Universitätsjubiläen regt zu einer vergleichenden Betrachtung an. Mögliche Fragestellungen betreffen die jeweilige Selbstinszenierung, die Abgrenzung zur ebenfalls jubilierenden „Konkurrenz“ und das daraus ableitbare unterschiedliche Selbstverständnis. Ferner bieten sich Konfliktthemen und eventuelle gemeinsame Aktivitäten sowie Unterschiede und Gemeinsamkeiten im Umgang mit der Geschichte der jeweils eigenen Organisation an. Der zweite Teil, der sich mit dem 200-Jahr-Jubiläum der TU Wien befasst, wird auch diese Aspekte berücksichti-

1 Dieses Ziel wurde nicht erreicht, das Projekt ist bis dato nicht abgeschlossen, weshalb es nunmehr nur noch den Namen ,univercity“ führt. Siehe TU Wien: TU Univercity. https://www.tu wien.at/tu-wien/campus/tu-univercity (17.12.2020).

2 Zur Bedeutung von Jubiläen für die Identifikationsbildung und Selbstinszenierung von Universitäten vgl. unter anderem Müller, Winfried: Die inszenierte Universität. Historische und aktuelle Perspektiven von Universitätsjubiläen. In: Jubiläum. Literatur- und kulturwissenschaftliche Annäherungen. Hrsg. von Franz M. Eybl, Stephan Müller u. Annegret Pelz. Göttingen 2018 (Schriften der Wiener Germanistik 6). S. 77-97.

3 Vgl. dazu eine erste Übersicht von Mikoletzky, Juliane u. Paulus Ebner: Einleitung. In: Dies.: Die Geschichte der Technischen Hochschule in Wien 1914-1955. Teil 1: Verdeckter Aufschwung zwischen Krieg und Krise (1914-1937). Wien/Köln/Weimar 2016 (Technik für Menschen. 200 Jahre Technische Universität Wien 1). S. 8-14. 
gen. Der Fokus liegt zwar auf den Jubiläumsfeierlichkeiten der TU Wien, doch sollen vergleichend verschiedene mediale Präsentationsformen wie die Jubiläums-Webseiten und sonstige Events der drei jubilierenden Universitäten herangezogen werden. Allerdings zeigte sich bei der Recherche, dass gerade elektronische Präsentationen, auch sehr aufwendig konzipierte, recht kurzlebig und heute meist nur noch schwer auffindbar sind. Als nachhaltiger erweisen sich die drei publizierten Festschriften. Sie sollen daher ausführlicher kommentiert werden, da sie mit ihren sehr unterschiedlichen Konzeptionen gute Einblicke in die jeweiligen Jubiläumskulturen und Auffassungen von „Universitätsgeschichte“ erlauben. Zugleich reflektieren sie auch die Funktion der jeweiligen Universitätsarchive.

\section{Traditionen des Feierns und der Erinnerung an der TU Wien: Frühere Bestandsjubiläen}

Die Etablierung von Gedenktraditionen ging für die TU Wien und ihre Vorgängerinstitutionen, das Polytechnische Institut in Wien beziehungsweise die Technische Hochschule in Wien, nicht ohne Schwierigkeiten vonstatten. Die Konstellation mehrerer gleichzeitig stattfindender Hochschuljubiläen hat sie stets begleitet und dazu geführt, dass es in Jubiläumsjahren jedes Mal einen Wettbewerb um öffentliche Aufmerksamkeit und auch um Ressourcen gab. Vor allem die übermächtige Konkurrenz der Universität Wien erwies sich von Beginn an als Hypothek. Dies zeigte sich bereits beim ersten Anlauf zu einer für das Jahr 1865 geplanten Feier anlässlich des 50-jährigen Bestandsjubiläums des Wiener Polytechnischen Instituts. Damals befanden sich die Polytechnischen Institute der Habsburgermonarchie, ähnlich wie die österreichischen Universitäten um die Wende zum 21. Jahrhundert, in einem Reformprozess, der ihre Organisationsstruktur modernisieren und an die Erfordernisse der sich entwickelnden technischen Wissenschaften anpassen sollte. Für das Wiener Polytechnische Institut mündeten diese Bemühungen 1865 in ein neues Organisationsstatut, und sie könnten den Entschluss für eine Jubiläumsfeier durchaus mit initiiert haben. ${ }^{4}$

4 Zur Reform des Wiener Polytechnischen Instituts vgl. Mikoletzky, Juliane:Vom Polytechnischen Institut zur Technischen Hochschule. Die Reform des technischen Studiums in Wien, 1850 - 1875. In: Mitteilungen der Österreichischen Gesellschaft für Wissenschaftsgeschichte 15 (1995). S. 79 100. 
Bereits 1861 nahm ein eigens gegründetes Komitee Vorarbeiten zu einem Festprogramm auf. ${ }^{5}$ Das Professorenkollegium konnte sich bald auf den 6. November 1815, den Tag der erstmaligen Aufnahme von Vorlesungen am Institut, als Bezugstermin für die Jubiläumsfeierlichkeiten einigen. Die Programmplanung enthielt schon alle wesentlichen Elemente, die sich auch bei späteren Jubiläen finden sollten. Für den eigentlichen Festtag sah sie nach einem Gottesdienst für die katholischen Angehörigen des Instituts in der benachbarten Karlskirche einen Festempfang mit Festessen vor, eventuell eine sogenannte „Liedertafel“. Außerdem sollte ein Porträt des ersten Direktors des Polytechnischen Instituts, Johann Joseph Prechtl, präsentiert werden. Vorgesehen waren weiterhin auch die Prägung einer Denkmünze, eine Reihe von materiellen Zuwendungen (Zubau eines kleinen Studien-Observatoriums, die Etablierung eines Reisestipendien-Fonds für Studenten) und eine Festschrift. Die Abfassung der Festschrift wurde bewusst keinem „Techniker“, sondern dem Professor für Geschäftsstil, Zivilgesetzkunde und Handelsgeographie Anton Langner (1816-1879) übertragen. Ein erhaltenes Exposé zeigt, dass sich Langner in Vorbereitung seiner Aufgabe auch Inspirationen bei bereits erschienenen Festschriften vergleichbarer Institutionen gesucht hatte, so bei jenen der Polytechnischen Institute in Prag (1856) und Graz (1861) sowie bei der Gedenkschrift zum 25-jährigen Bestandsjubiläum der Polytechnischen Schule in Hannover (1856). ${ }^{6}$ Das spricht dafür, dass es um die Mitte des 19. Jahrhunderts bereits Ansätze zu einer Identitätsbildung der als Typus ja noch jungen Polytechnischen Institute in der Monarchie beziehungsweise im deutschsprachigen Raum gab.

Trotz dieser bereits weit gediehenen Vorbereitungen sagte das Professorenkollegium des Instituts am 11. Mai 1864 sämtliche Feierlichkeiten ab. Zu diesem Zeitpunkt war absehbar geworden, dass es nicht gelingen würde, die notwendigen Budgetmittel für das geplante Programm aufzubringen. Mit einer bescheideneren Feier fürchtete man jedoch, wie es Anton Schrötter, Professor für Allgemeine Chemie und Generalsekretär der kaiserlichen Akademie der Wissenschaften, in einer Sitzung des Festkomitees vom 9. Mai 1864 formulierte, in der Öffentlichkeit keine hinreichende Aufmerksamkeit mehr zu finden, da zugleich die Universität Wien im August 1865 mit großem Aufwand ihr 500-Jahr-Jubiläum

5 Die Originalakten des Festkomitees haben sich im Archiv der TU Wien leider nicht erhalten, die folgende Darstellung stützt sich daher weitgehend auf Neuwirth, Joseph (Hrsg.): Die k.k. Technische Hochschule in Wien 1815-1915. Gedenkschrift. Wien 1915. S. 277-280.

6 Das Exposé befindet sich im Personalakt von Karl Langner, Archiv der TU Wien (AT TUWA), Personalakt Karl Langner. Das Manuskript der Festschrift, die 1864 bereits nahezu fertiggestellt gewesen sein soll, hat sich leider nicht erhalten; vgl. zur Jubiläumskultur von Polytechnika im 19. Jahrhundert den Beitrag von Anton F. Guhl in diesem Band. 
begehen würde. ${ }^{7}$ Diese Absage war also das Ergebnis einer langfristig-strategisch gedachten Entscheidung: Dem Anspruch des Wiener Polytechnischen Instituts auf Behauptung als größte höhere technische Bildungsanstalt der Monarchie und auf Gleichrangigkeit mit der Universität Wien sollte durch eine misslungene Selbstpräsentation kein Abbruch getan werden.

Erfolgreicher war die gleichzeitig gestartete Initiative einer studentischen „Gegen-Festschrift“: 1861 begehrte der damals gerade 20-jährige Hörer am Wiener Polytechnischen Institut Wilhelm Exner vom Professorenkollegium Zugang zu den Institutsakten, um daraus eine kritische Geschichte der Einrichtung zu verfassen. Dies wurde ihm jedoch verwehrt, da die Professoren keine „offiziöse“ Publikation neben der geplanten Festschrift wünschten. Exner veröffentlichte trotzdem eine Darstellung der bisherigen Geschichte des Instituts, und zwar noch im selben Jahr. ${ }^{8}$ Dabei stützte er sich offenbar hauptsächlich auf gedruckte Quellen. Er griff auch den bereits seit Ende der 1840er Jahre geführten Diskurs um eine notwendige Reform des Technikstudiums auf (Exner war 1861 Mitglied einer Studentendeputation, die beim zuständigen Unterrichtsminister Schmerling Verbesserungen einforderte) und endete mit einer Reihe kritischer Fragen zum Zustand des Instituts, deren Beantwortung er wohlweislich seinen Lesern überlassen wollte. Inhaltlich unterschied sich seine Position nur wenig von jener, die auch das Professorenkollegium einnahm. Eine offizielle Reaktion auf die Schrift ist nicht bekannt, vielleicht auch, weil Exner inzwischen das Institut bereits verlassen hatte. In der 100-Jahr-Festschrift wird er jedenfalls lobend als ,erster Geschichtsschreiber“ der Hochschule erwähnt. ${ }^{9}$

Auch die folgenden Jubiläumstermine standen unter keinem guten Stern: Die großangelegten Vorbereitungen für eine 100-Jahr-Feier der nunmehrigen „k. k. Technischen Hochschule in Wien“ im Jahr 1915 fielen weitestgehend dem

7 Vgl. dazu Neuwirth, Hochschule (wie Anm. 5), S. 279f.; siehe auch AT TUWA, Protokolle der Sitzungen des Professorenkollegiums des Polytechnischen Instituts in Wien: Protokoll der Sitzung vom 11. Mai 1864, TOP X.

8 Exner, Wilhelm Franz: Das k.k. polytechnische Institut in Wien. Seine Gründung, seine Entwickelung und sein jetziger Zustand. Wien 1861; Exner (1882-1897) wurde 1868 Professor an der Forstakademie Mariabrunn, ab 1875 ordentlicher Professor der allgemeinen mechanischen Technologie und des forstlichen Bau- und Maschineningenieurswesens an der neu gegründeten Wiener Hochschule für Bodenkultur. Als liberaler Reichstagsabgeordneter und seit 1905 Sektionschef im Handelsministerium machte er sich besonders um die Etablierung des Technischen Versuchswesens in Österreich verdient. Auch an der Gründung des heutigen Technischen $\mathrm{Mu}$ seums in Wien war er maßgeblich beteiligt.

9 Neuwirth, Hochschule (wie Anm. 5), S. 280. 
Ersten Weltkrieg zum Opfer - tatsächlich realisiert wurde nur eine Festschrift. ${ }^{10}$ Der repräsentative Band im Quartformat umfasst 700 Seiten, einem Teil der Auflage sind 18 Lichtdrucke beziehungsweise Heliogravüren mit Porträts von Professoren beigegeben. Die Ausstattung war unmittelbar durch die Kriegsereignisse beeinträchtigt: Ursprünglich sollte der Entwurf des Einbandes durch einen Wettbewerb unter den Hörern der Bauschule ermittelt werden - zum Zeitpunkt der Entscheidung waren jedoch die vier aussichtsreichsten Bewerber bereits zum Kriegsdienst eingezogen. Daher übernahm der Architekt und Professor an der TH Wien, Max Fabiani, die Einbandgestaltung. Wohl aus Kostengründen wurde nur ein sehr kleiner Teil der Auflage als „Prachtausgabe“ mit Lederrücken beziehungsweise mit hochwertigem Leineneinband hergestellt. Diese Exemplare waren überwiegend als Geschenk für Mitglieder des Kaiserhauses, Minister und andere hochrangige Würdenträger sowie für die Rektoren befreundeter Hochschulen vorgesehen. Der bei weitem größte Teil der 800 gedruckten Exemplare erhielt einen einfachen Leineneinband oder wurde überhaupt nur in Broschur gebunden. Damit sollte auch Studierenden ein Erwerb der Festschrift ermöglicht werden. Inhaltlich behandelte ihr Herausgeber, der positivistisch geschulte Kunsthistoriker Joseph Neuwirth, in einem historischen Teil ausführlich und sehr quellennah die Gründungsgeschichte und weitere organisatorische Entwicklung der Hochschule bis zur Jahrhundertwende. Das schloss eine Geschichte der Studierenden sowie weiterer Einrichtungen und einen umfangreichen statistischen Anhang ein. Die Geschichte der Lehrkanzeln (Institute gab es damals noch nicht) wurde, geordnet nach den Fachschulen, von den jeweiligen Lehrkanzelvorständen in ähnlicher Form dargestellt. Strukturell ähnelte diese Festschrift durchaus dem Entwurf, den Langner seinerzeit für 1865 vorgelegt hatte. Verstärkt wurde eine Gedenktradition, die stark auf die Gründungsphase und die frühe Geschichte zu Beginn des 19. Jahrhunderts fokussierte, als das Polytechnische Institut tatsächlich für einige Jahrzehnte eine europaweite Modellfunktion als moderne technische Lehranstalt hatte.

Die Feier des 125-Jahr-Jubiläums 1940 stand im Schatten des Zweiten Weltkriegs, war aber zugleich auch Gegenstand nachdrücklicher Interventionen verschiedener politischer Stellen. Im Herbst 1939 waren entsprechende Vorbereitungen aufgrund des Kriegsbeginns zunächst abgesagt worden. ${ }^{11}$ Im März 1940 nahm die Hochschule jedoch Planungen für eine Feier in bescheidenem Rahmen wieder auf. Das für die Bewilligung zuständige Reichsministerium für Wissen-

10 Neuwirth, Hochschule (wie Anm. 5); zum Folgenden siehe AT TUWA, Sonderlegung 100-JahrJubiläum.

11 Vgl. für das Folgende AT TUWA, Sonderlegung 125-Jahr-Jubiläum; siehe dazu auch den Beitrag von Martin Göllnitz und Paula Rilling in diesem Band. 
schaft, Erziehung und Volksbildung (REM) genehmigte nicht nur zusätzliche Mittel, sondern es verlangte auch eine wesentlich aufwendigere Gestaltung, insbesondere was die Einladung von hochrangigen politischen Gästen betraf. Die angeblich besondere Bedeutung der TH Wien für den „Südosten“ Europas sollte als Vorwand für die Propagierung der „Südost-Politik“ des Reiches dienen. Das lag auch im Interesse des Wiener Gauleiters und Reichsstatthalters Baldur von Schirach, der ebenfalls seine Unterstützung zusagte. Daher musste die Hochschule ab September 1940 eiligst mit den Planungen beginnen, wobei bis in den eigentlichen Festakt hinein ständig Wünsche und Anordnungen sowohl von Seiten des REM als auch von lokalen militärischen und Parteistellen geäußert wurden. Das Programm für die drei Tage vom 6. bis 8. November umfasste schließlich einen Festakt in Anwesenheit von Unterrichtsminister Bernhard Rust, der Minister Todt, Dorpmüller und Schwerin von Krosigk, des Reichsstatthalters von Schirach sowie allerlei lokaler NS-Prominenz, eine Reihe von „Ehrenvorträgen“ prominenter Techniker, einige Empfänge sowie ein kleines touristisches Programm mit einem Abend in der Wiener Staatsoper als Abschluss. Außerdem erschien, allerdings mit zweijähriger Verspätung, eine sehr schmale, eher chronikalisch angelegte Festschrift, verfasst von dem seit langem schon an der Geschichte der Hochschule interessierten Professor für Mechanik Alfred Lechner. ${ }^{12}$ Ehrendoktorate konnten keine vergeben werden, da die Vorschläge dafür an das REM zur Genehmigung angeblich zu spät eingelangt waren. Entsprechend nüchtern klingen die Abschlussberichte der Mitglieder des Festkomitees, die einen gewissen Unmut über die doch weitgehende Fremdbestimmung der Jubiläumsfeierlichkeiten erkennen lassen.

Umso mehr sollte 1965 das 150-Jahr-Jubiläum mit allem gebührenden Aufwand begangen werden, wozu wiederum die Erarbeitung einer, nunmehr dreibändigen, Festschrift gehörte. Die Konkurrenz durch die parallel stattfindenden Jubiläumsfeiern der Universität Wien (600 Jahre) und der Veterinärmedizinischen Hochschule (200 Jahre) versuchte man zunächst über Kooperationsangebote zu neutralisieren. Eine im Vorfeld 1959 in der Österreichischen Rektorenkonferenz diskutierte gemeinsame Feier der drei Jubel-Hochschulen im Rahmen einer „Akademischen Woche“13 erwies sich jedoch letztlich als nicht mehrheitsfähig. So feierte weiterhin jede Institution für sich.

12 Lechner, Alfred: Geschichte der Technischen Hochschule in Wien (1815-1940). Hrsg. von der Technischen Hochschule in Wien. Wien 1942.

13 Vgl. AT TUWA, Rektoratsakten, RZl. 1570/1959, Österreichische Rektorenkonferenz: Protokoll der Sitzung vom 20. Juni 1959, TOP 2. 
Diese erste „richtige“, weil endlich ohne äußere Einschränkungen und nach eigenen Vorstellungen gestaltete Jubiläumsfeier der TH in Wien setzte Maßstäbe, mit denen sich später auch die Organisator*innen der 200-Jahr-Feiern 2015 auseinandersetzten mussten. ${ }^{14}$ Die Motive des Vorbereitungsausschusses zum Jubiläum 1965 lassen sich dem Dokumentationsband der Festschrift entnehmen. Die Intention war, zu zeigen, ,wie umfassend und harmonisch Wissenschaft und Technik von heute sind“, und damit die Positionierung der angewandten Naturund Ingenieurwissenschaften als ,gleichwertig“ mit den übrigen Wissenschaften (und damit natürlich auch die Gleichwertigkeit ihrer Trägerinstitutionen, der Technischen Hochschulen, mit den klassischen Universitäten) zu behaupten: „Längst sind die - einst künstlich errichteten - Schranken zwischen den an den Universitäten und an den technischen Hochschulen gepflegten Wissenschaften gefallen. “15 Damit wurde Bezug genommen auf eine vor allem in Deutschland seit dem späten 19. Jahrhundert geführte Diskussion um die Rangordnung der tertiären Bildungsinstitutionen, in der Technische Hochschulen, Ingenieure und Techniker die Gleichstellung mit den klassischen Universitäten und ihren Absolventen einforderten. Allerdings war diese Kluft in der Habsburger Monarchie und in der Republik Österreich bei weitem nicht so scharf ausgeprägt wie im Deutschen Reich - was wohl nicht zuletzt dem Gründer des Wiener Polytechnischen Instituts, Johann Joseph Prechtl, und dem von ihm energisch vertretenen Konzept der Gleichrangigkeit des Instituts als einer „universitas scientiarum technicarum“ zu verdanken war. Es sei hier daran erinnert, dass ähnliche Überlegungen bereits bei den Vorbereitungen zum geplanten Jubiläum 1865 im Hintergrund standen.

1965 ging es also um ein Statement der Selbstbehauptung der „Technik“ (als Institution und als Wissenschaftsfeld) und ihrer Fachvertreter, dies auch vor dem Hintergrund des Kalten Krieges und dem von ihm ausgelösten Wettlauf in der Technikentwicklung. Im selben Kontext begann etwa zeitgleich - nicht nur in Österreich - die Diskussion um eine Hochschulreform und einen deutlichen Ausbau der natur- und technikwissenschaftlichen Fächer, in Österreich auch um eine Verbesserung und Ausweitung der staatlichen Forschungsförderung. ${ }^{16}$ In der

14 Vgl. für das gesamte Festprogramm die ausführliche Dokumentation in Sequenz, Heinrich (Hrsg.): 150 Jahre Technische Hochschule in Wien, 1815-1965. Bd 3.: Verlauf der Hundertfünfzigjahrfeier. Wien 1967.

15 Kresser, Werner, Geleitwort. In: Sequenz, 150 Jahre (wie Anm. 14), S. 5 f.

16 In Österreich führte sie mit dem Universitätsorganisationsgesetz (UOG) 1975 zu einer völligen, rechtlichen und sprachlichen Gleichstellung der nunmehr in „Technische Universitäten“ umbenannten Hochschulen technischer Richtung mit den klassischen Universitäten. Zur For- 
Gestaltung der Jubiläumsfeierlichkeiten der TH Wien schlugen sich diese Intentionen in mehrfacher Hinsicht nieder: Neben den „klassischen“ Elementen des Feierns, wie Festakten und sonstigen gesellschaftlichen Ereignissen (Empfänge, Festaufführungen in der Staatsoper und im Burgtheater), der Verleihung akademischer Ehrungen und letztlich auch der Erstellung einer Festschrift umfasste das Programm auch Elemente einer „Leistungsschau“ der (österreichischen) Technik in Form von Ausstellungen und einer Serie wissenschaftlicher Fachvorträge. Nicht zuletzt gehörte dazu auch eine detaillierte Liste der anlässlich des Jubiläums eingegangenen Subventionszusagen staatlicher Stellen und von Spenden vorwiegend industrieller Gönner. Dazu kamen mediale Selbstpräsentationen der TH Wien, etwa in Sondernummern der Österreichischen Hochschulzeitung und in der Zeitschrift des Österreichischen Ingenieur- und Architektenvereins (ÖIAV) oder durch einen Fotowettbewerb. Die Enthüllung von Gedenktafeln und Büsten namhafter Techniker und Hochschulangehöriger in der Aula des Hauptgebäudes ist hier ebenso zu nennen wie die Schaffung neuer Talare für die akademischen Funktionäre, die dann während der Feierlichkeiten ausgiebig zum Einsatz kamen.

Die erwünschte „Gravitas“ wurde hergestellt durch Bezugnahmen auf die Geschichte der Hochschule, insbesondere auf die Gründungsperiode des Polytechnischen Instituts, und durch die Aktivierung von weiteren Traditionselementen, wie sie bereits für 1865 geplant gewesen waren. Dazu gehörte die Kranzniederlegung am Grab des Institutsgründers und ersten Direktors Prechtl auf dem Wiener Zentralfriedhof am 8. November ebenso wie der (katholische) Festgottesdienst in der Karlskirche. In der Festschrift, die von ihrer Konzeption her die umfangreiche Darstellung aus 1915 ergänzen sollte, widmete sich einer der beiden inhaltlichen Bände der „Geschichte und Ausstrahlung“ der TH Wien und den Biographien prominenter Angehöriger, der zweite umfasste Beiträge zur Baugeschichte sowie zur Geschichte der einzelnen Institute und ihrer bisherigen wie aktuellen Leistungen. Eigene Kapitel waren der Hochschulbibliothek und der Österreichischen Hochschülerschaft als Studierendenvertretung gewidmet. Der dritte Band diente der Dokumentation des Festgeschehens. Zusätzlich erstellte die Hochschülerschaft eine eigene Jubiläumspublikation, in der sie die Geschichte der Studierenden nachzeichnete - diesmal nicht in Konkurrenz, sondern durchaus als erwünschte Ergänzung zur Festschrift. ${ }^{17}$ Diese Einbeziehung der Studierenden ist charakteristisch für das traditionell eher konsensorientierte Klima an der Hochschule, das die Hörer (erst ab 1919 auch Hörerinnen) schon frühzeitig als

schungsförderung vgl. Pichler, Rupert, Michael Stampfer u. Reinhold Hofer: Forschung, Geld und Politik. Die staatliche Forschungsförderung in Österreich 1945-2005. Innsbruck 2007.

17 Österreichischen Hochschülerschaft an der technischen Hochschule Wien (Hrsg.): 150 Jahre Technische Hochschule Wien. Eine Geschichte ihrer Studenten. Wien [1965]. 
Gesprächspartner einbezog. Dass es sich bei der Gestaltung des Festprogramms in überraschend geringem Maße um eine auf die TH zugeschnittene Programmierung handelte, sondern vielmehr um die Realisierung eines zeittypischen Musters, zeigt ein Vergleich mit den nur wenige Monate früher angesetzten Jubiläumsfeierlichkeiten der Universität Wien, die bis ins Detail hinein zahlreiche Übereinstimmungen aufwiesen. ${ }^{18}$

$\mathrm{Zu}$ den aus heutiger Sicht problematischen Elementen der Feiern gehörte der Umgang mit der jüngeren Vergangenheit der Hochschule: Die Organisation des Festprogramms war ausgerechnet in die Hände des letzten NS-Rektors der TH Wien, Heinrich Sequenz (1895-1987), gelegt worden. Nach Kriegsende entlassen, seit 1954 als Professor wieder voll reaktiviert, brachte dieser eine Reihe von sehr traditionellen Elementen in die Feiern ein, so einen studentischen Fackelzug am Vorabend des Gründungstages und die Enthüllung eines Denkmals für die im Zweiten Weltkrieg gefallenen Angehörigen der Hochschule in der Aula des Hauptgebäudes, gegenüber der Gedenktafel für die Gefallenen des Ersten Weltkriegs. Zwar wurden in dem zugehörigen „Ehrenbuch“ neben den Namen der Gefallenen auch jene (wenigen) der „Opfer des Bombenkrieges und des Widerstandes“ verzeichnet. Auf die während der NS-Zeit vertriebenen und ermordeten Angehörigen der TH Wien wurde jedoch mit keiner Silbe Bezug genommen. Auch in den Beiträgen zur Festschrift wurde die Rolle der TH Wien zwischen 1938 und 1945 kaum berücksichtigt, allenfalls Bombenschäden an einzelnen Gebäuden wurden erwähnt, die politischen Verhältnisse und das Schicksal der verfolgten Angehörigen fanden jedoch kaum, und wenn, dann in verharmlosenden und beschönigenden Worten Berücksichtigung.

In dieser Hinsicht war die TH Wien freilich kein Einzelfall, denn auch die Festprogramme und Festschriften anderer österreichischer Hochschulen aus dieser Zeit sparten die NS-Zeit weitestgehend aus. Das änderte sich erst langsam mit der Errichtung eines Instituts für Zeitgeschichte an der Universität Wien 1966 und insbesondere im Zuge der „Waldheim-Debatte“ 1986 und des „Gedenkjahrs“ 1988, als sich die österreichischen Universitäten erstmals intensiver professionell mit ihrer eigenen NS-Vergangenheit zu beschäftigen begannen. ${ }^{19}$ An der TU Wien schlug sich dies allerdings bei der Gestaltung des 175-Jahr-Jubiläums 1990 noch nicht nieder. Eine Festschrift gab es damals nicht, wohl aber historisch orientierte

18 Vgl. dazu die Beschreibung der Feierlichkeiten an der Universität Wien bei Schmidt-Lauber, Brigitta: Die (sich) feiernde Universität. In: Eybl/Müller/Pelz, Jubiläum (wie Anm. 2), S. 99-114, hier S. 102-106.

19 Vgl. dazu als eine der frühesten Publikationen Heiß, Gernot [u.a.] (Hrsg.): Willfährige Wissenschaft. Die Universität Wien 1938-1945. Wien 1989. 
Tagungen und Publikationen, die Person und Werk des Gründers Johann Joseph Prechtl betrafen und somit neuerlich stark auf den „Gründungsmythos“ setzten. ${ }^{20}$

Signifikant für alle bis in die 1990er Jahre erarbeiteten Darstellungen zur Geschichte der TU Wien und ihrer Vorgängerinstitutionen ist, dass sie ausschließlich von Angehörigen des Hauses, und das heißt: von historisch interessierten, ausgebildeten Ingenieuren, Architekten, fallweise von Kunsthistorikerinnen verfasst wurden, nicht von professionellen Historiker^innen. Das liegt einerseits daran, dass es an der TU Wien wie an allen österreichischen Hochschulen technischer Richtung, anders als an ihren deutschen Schwesterinstitutionen, weitgehend an der entsprechenden fachhistorischen Kompetenz mangelt, da dort bis heute die geistesund sozialwissenschaftlichen Disziplinen einschließlich der Geschichtswissenschaft institutionell nicht verankert sind. Andererseits sind die Technikgeschichte und auch das Interesse an einer Geschichte der technischen Bildungsinstitutionen derzeit an den klassischen Universitäten in Österreich kaum vertreten. Dies wiegt umso schwerer, als auch wissenschaftlich betreute Universitätsarchive an diesen Hochschulen erst seit den letzten Jahrzehnten des 20. Jahrhunderts eingerichtet wurden.

An der TU Wien geschah dies Ende der 1970er Jahre. Bis 1991 wurde das Archiv von einem wissenschaftlichen Beamten und ausgebildeten Architekten aufgebaut und geleitet. Ab 1992 wurde mit der Verfasserin erstmals eine professionelle Historikerin angestellt, die Leitung übernahm jedoch wiederum ein Ingenieur, dem die Verfasserin 2001 nachfolgte. Erst seitdem verfügt das Archiv über zwei Stellen für ausgebildete Historiker`innen als Leiter`in und Mitarbeiter^in. Seit Anfang der 1990er Jahre hat sich das Archiv durch Ausstellungen, Publikationen und Forschungsprojekte zur Geschichte der TU Wien, auch für die Zeit des Nationalsozialismus, als Ansprechpartner für alle Fragen zur Geschichte der Universität positioniert. Dies war ein wesentlicher Ansatzpunkt für die Einbeziehung des Archivs in die Gestaltung der 200-Jahr-Feier 2015.

\section{Die 200-Jahr-Feier der TU Wien 2015: Zwischen Tradition und Neuinszenierung}

Die Ausganglage für die Planungen der Feier des 200-Jahr-Jubiläums der TU Wien war einerseits geprägt durch die absehbare Aufmerksamkeits-Konkurrenz durch die zeitgleichen Jubiläen der Universität Wien und der Veterinärmedizinischen

20 Vgl. Hantschk, Christian (Hrsg.): Johann Joseph Prechtl. Sichtweisen und Aktualität seines Werkes, anlässlich 175 Jahre Technische Universität Wien. Wien/Köln 1990. 
Universität Wien, andererseits dadurch, dass alle drei Institutionen eine Phase tiefgreifender institutioneller Umgestaltung hinter sich hatten beziehungsweise sich weiterhin in einem Restrukturierungsprozess befanden. Unmittelbar relevant für die Durchführung der Jubiläumsfeiern war insbesondere der neue Finanzierungsmodus: Neben einer Basisabgeltung durch die öffentliche Hand aufgrund von Leistungsvereinbarungen waren die Universitäten nun für ihre weitere Ausgestaltung, wissenschaftliche Profilentwicklung und für sonstige Aktivitäten im Rahmen ihrer „Third Mission“ auf das kompetitive Einwerben von externen Fördermitteln angewiesen.

Im universitären Alltag besonders spürbar waren die tiefgreifenden organisatorischen Umstrukturierungen, nachdem die interne Gliederung nicht mehr von den Unterrichtsbehörden vorgegeben wurde, sondern von den Hochschulen selbst gestaltet und entwickelt werden sollte. An der TU Wien wurden aus bisher fünf jetzt acht Fakultäten gebildet, Institute zu größeren Einheiten zusammengefasst, administrative Dienststellen nach unterschiedlichen Funktionskonzepten den Aufgabenbereichen des Rektors/der Rektorin oder der Vizerektor`innen zugewiesen. An der Universität Wien erfolgte unter anderem die Ausgliederung der mathematisch-naturwissenschaftlichen Fächer aus der Philosophischen Fakultät in eine Reihe eigener Fakultäten. Anstelle der ehemaligen Medizinischen Fakultät wurde eine eigenständige „Medizinische Universität Wien“ geschaffen. Diese Umgestaltungsprozesse warfen nach innen Identifikationsfragen für die Universitätsangehörigen auf. Nach außen ergab sich daraus ein Bedarf nach Gestaltung eines neuen Selbstverständnisses der drei Wiener Universitäten als wissenschaftlicher Einrichtungen, nach neuen Formen der Selbstinszenierung und entsprechender Kommunikation. Wegen der Anforderungen an eine „leistungs-“ oder „exzellenz-“orientierte Universitätsfinanzierung wurden gesellschaftliche Relevanzbehauptungen zu essenziellen Argumenten in diesem Diskurs. Es kam hinzu, dass zu dem Zeitpunkt, als die Universitäten mit ihren Jubiläums-Planungen beginnen mussten, die Nachwirkungen der Finanzkrise von 2008 noch deutlich zu spüren waren und allerseits (anders als 1965) zunächst wenig Begeisterung bestand, sich auf aufwändige Feierlichkeiten einzulassen. ${ }^{21}$

21 Da die Akten zum 200-Jahr-Jubiläum der TU Wien noch nicht zugänglich sind, konnte sich die folgende Darstellung nur auf die vorhandenen Publikationen stützen; dazu gehörten neben der einstigen Jubiläums-Webseite TU Wien: TU 200. 1815-2015. 200 Jahre Zukunft. http://www.tu200. at (17.12.2020) auch einige Ausgaben der Mitgliederzeitschrift „freihaus“ der TU Wien, unter anderem die Nr. 36 mit einem Rückblick auf das Jubiläumsjahr: frei.haus. Online-Magazin für Mitarbeiter_innen der TU Wien: 200 Jahre TU Wien. Nachschau. https://freihaus.tuwien.ac.at/ 200-jahre-tu-wien-nachschau/ (17.12.2020), sowie auf die Erinnerungen der Verfasserin als Mitglied des Jubiläumskomitees. 
Daher nahmen die drei Institutionen, insbesondere die TU Wien und die Universität Wien, im Vorfeld frühzeitig Gespräche auf, um Bereiche eventueller Ressourcenkonkurrenzen aufzuspüren und mögliche Synergieeffekte zu finden. Es kam jedoch auch diesmal kaum zu wirklichen Kooperationen: So erfolgte die Teilnahme aller drei Universitäten an übergreifenden Wissenschafts-Events, etwa am „Wiener Forschungsfest“, mit jeweils eigenem Programm. Beim Neujahrskonzert im Wiener Musikverein des Jahres 2015 fand eine Art Aufteilung zwischen TU Wien und Universität Wien statt: Die Musikauswahl setzte vor allem auf die Strauss-Brüder, die beide das Polytechnische Institut besucht und seinen Hörern auch einige Kompositionen gewidmet hatten, während die Aufnahmen für die Pauseneinlage im Innenhof des Hauptgebäudes der Universität Wien gedreht worden waren. ${ }^{22}$

Von der TU Wien selbst wurde das Jubiläum im Wesentlichen als klar strukturiertes PR-Projekt geplant. Die erarbeiteten Strategien sollten einerseits ein Zusammengehörigkeitsgefühl von Mitarbeiter*innen und Studierenden und ihre aktive Identifikation nicht nur mit dem eigenen Institut oder der eigenen Fakultät, sondern auch mit der Gesamtuniversität unterstützen. Ziel war eine Stärkung der „Corporate Identity“ nach innen. Daher wurden auch alle Gruppen der Universität in die Vorbereitung der Festlichkeiten und die entsprechenden Gremien und Jubiläumskomitees eingebunden. Nach außen sollte die TU Wien durch ein neues „Corporate Image“ als hervorragende Forschungseinrichtung und als „Forschungsuniversität“ positioniert werden. Dadurch sollte die Attraktivität der Universität einerseits für Studierende und hochqualifizierte Forscher`innen, andererseits für Kooperationen mit der Industrie und für ein entsprechendes Fundraising erhöht werden. Zukunftsorientierung war angesagt. Die Ausgangslage für entsprechende Argumentationen war inzwischen eine deutlich andere als 50 Jahre zuvor. Zentrale Anliegen der Selbstdarstellung der „Technik“ aus der zweiten Hälfte des 20. Jahrhunderts waren kein Thema mehr. Die Position der Ingenieur- und Technikwissenschaften war inzwischen mehr als gefestigt, in der Defensive befanden sich eher die Geisteswissenschaften. Die verbreitete TechnikSkepsis, wie sie vor allem die 1980er und 1990er Jahre prägte, hatte ebenfalls an Bedeutung verloren. Vielmehr waren die Technikwissenschaften, insbesondere die aufstrebenden Informationswissenschaften, und die von ihnen erhoffte Innovationskraft in aller Munde.

22 Zum Programm siehe Austria-Forum: Neujahrskonzert der Wiener Philharmoniker 2015. https://austria-forum.org/af/AustriaWiki/Neujahrskonzert_der_Wiener_Philharmoniker_2015 (2.2.2021). 
Damit war zugleich klar, dass die ungebrochene Fortführung früherer Festtraditionen und eine historische Selbstvergewisserung gerade nicht im Vordergrund der Feierlichkeiten stehen sollten. Die eigene Geschichte wurde nicht (mehr) als geeignete Vermarktungsressource wahrgenommen. Ausnahmen wie die Präsentation eines „virtuellen Fallschirmsprungs“ durch die 200 Jahre der TUGeschichte auf dem Wiener Forschungsfest bestätigen nur, dass „Geschichte“ allenfalls als Illustrationsmaterial gesehen wurde, ohne einen Vermittlungs- oder Erkenntnisanspruch. ${ }^{23}$ Das professionell entwickelte Jubiläums-Design (auch dies ein Merkmal der Jubiläen des Jahres 2015), das sämtliche Kommunikationskanäle von der Website über alle Drucksorten, Publikationen, Merchandisingund Werbeartikel bespielen sollte, war betont unkonventionell und modern, die verwendeten Slogans suggerierten Dynamik: „200 Jahre Zukunft“; „Wir gestalten die Zukunft der Technik“ (wobei der Begriff „Technik“ hier sowohl auf das wissenschaftliche und gesellschaftliche Handlungsfeld als auch auf eine gebräuchliche Kurzbezeichnung für die TU Wien anspielte). Auch aus budgetären Gründen erfolgte ein „Branding“ all jener Veranstaltungen, die im Laufe des Jahres 2015 ohnehin stattfinden sollten, wie Tagungen, Kongresse, die jährlichen Fakultätentage und nicht zuletzt der TU Ball, als „Jubiläumsveranstaltungen“. Damit wurde eine Ausdehnung der Jubiläumsfeierlichkeiten auf das ganze Jahr 2015 erreicht. Die Universität Wien verfolgte eine ähnliche Strategie. ${ }^{24}$ Spezielle Jubiläumsevents waren vor allem darauf ausgelegt, die Zukunftsfähigkeit der Technik zu demonstrieren: so etwa die Diskursreihe „TU Vision 2025+“"25, die Ausstellung der „Wiener Wunderkammer“, die ein Zusammenwirken von Kunst und Technik präsentierte ${ }^{26}$, oder der „Galaabend Technik“, ein großes Fundraising-Dinner, das von einer wissenschaftlichen „Leistungsschau“ der TU Wien begleitet war.

23 Vgl. dazu Aigner, Florian: Fliegen durch Raum und Zeit. TU Wien entwickelt virtuellen Skydive. https://www.tuwien.at/tu-wien/aktuelles/news/news/fliegen-durch-raum-und-zeit-tuwien-entwickelt-virtuellen-skydive/ (17.12.2020).

24 Schmidt-Lauber, Universität (wie Anm. 18), S. 106-110; zu den Jubiläumsplanungen der Universität Wien vgl. auch: Ash, Mitchell G.: Die Universitätsgeschichtsschreibung an der Universität Wien im Jubiläumsjahr 2015 - zwischen historischer Reflexion und Eventkultur. In: Universitätsgeschichte schreiben. Inhalte - Methoden - Fallbeispiele. Hrsg. von Livia Prüll, Christian George u. Frank Hüther. Mainz 2019 (Beiträge zur Geschichte der Universität Mainz Neue Folge 14). S. 221-239.

25 Siehe TU Vision 2025+. Ein strategisches Projekt der TU Wien. https://vision2025.tuwien.ac.at/ home_about/ (17.12.2020).

26 Vgl. dazu Schwinghammer, Susanne: Sehen und Staunen lernen. Die Wiener Wunderkammer 2015. https://www.tuwien.at/tu-wien/aktuelles/news/news/sehen-und-staunen-lernen-die-wie ner-wunderkammer-2015/ (17.12.2020); zum Konzept vgl. auch den Beitrag des Initiators der „Wunderkammer“: Überhuber, Christoph: Kunst, Wissenschaft und Technik. In: Die Technik und 
Traditionelle Fest-Konventionen wurden nicht gänzlich ausgelassen, waren aber eher nach „innen“ gerichtet und schienen in der öffentlichen Jubiläumsberichterstattung der TU Wien kaum auf. So hielt die Universitätsleitung an der Kranzniederlegung am Grab Prechtls ebenso fest wie an der Jubiläumsmesse in der Karlskirche, der Herausgabe einer Denkmünze und einer Sonderbriefmarke, während ein Gedenken an die Opfer der beiden Weltkriege schon mangels eines geeigneten Ortes ausfiel - die beiden Denkmäler waren im Zuge der Neugestaltung der Aula des Hauptgebäudes ersatzlos entfernt worden. Der eigentliche Festakt war in seiner Gestaltung ebenfalls eher traditionell geprägt. Auch die Entscheidung für die Herausgabe einer Festschrift gehörte zu diesen konventionelleren Jubiläums-Elementen. Damit stellte sich implizit doch die Frage nach einer historischen Reflexion. Es war jedoch der ausdrückliche Wunsch des Rektorats, entsprechend dem zukunftsbezogenen Gesamtkonzept des Jubiläumsjahrs möglichst keine umfassenden historischen Darstellungen der Geschichte der TU Wien vorzulegen und auch die bisher bei solchen Gelegenheiten üblichen Referenzen auf den „Gründungsmythos“ der TU Wien zu vermeiden. Das Archiv als einzige Einrichtung der TU Wien, die sich auch bisher schon mit der Geschichte der Universität professionell beschäftigt hatte, war dabei von Beginn an in die Überlegungen zu diesem Projekt einbezogen. Die Verfasserin als damalige Leiterin des Archivs war Mitglied des Jubiläumskomitees und wurde letztlich auch mit der Gesamtkoordination und Redaktion der Festschrift beauftragt. Unter Berücksichtigung der Vorgaben des Rektorats, der bereits vorhandenen älteren Festschriften sowie der verfügbaren personellen Ressourcen schlug die Verfasserin ein Konzept vor, das den Fokus auf die Betrachtung der seit dem letzten Jubiläum vergangenen 50 Jahre, die organisatorische Entwicklung der Universität, ihrer Einrichtungen und vor allem der dort betriebenen Forschung legen sollte. Wie bei den früheren Festschriften sollte die Darstellung durch Angehörige des Hauses erfolgen, unter inhaltlicher Verantwortung der jeweiligen Fakultäten. Dies schien wegen der starken Orientierung auf die Präsentation der Forschungsleitungen sachlich geboten, sollte aber auch einen gewissen Anreiz zur aktiven Einbindung der Fakultäten in ein gemeinsames Vorhaben bieten und damit zur Stärkung der Corporate Identity nach innen beitragen. Das Archiv bot seine Unterstützung bei der Beschaffung von historischen Unterlagen, Informationen und Bildmaterial an.

die Musen. Kunst und Kultur im Umfeld der Technischen Universität Wien. Hrsg. von Juliane Mikoletzky. Wien 2016 (Technik für Menschen. 200 Jahre Technische Universität Wien 14). S. 11-26. 
Die Beiträge sollten sich an eine allgemeine Leserschaft richten. Aus Gründen größerer Flexibilität bei der Fertigstellung wurde die Veröffentlichung nicht in einem oder mehreren umfangreichen Sammelbänden geplant, sondern es erfolgte eine Aufteilung auf eine größere Anzahl weniger voluminöser Einzelbände, die auch separat zu erwerben sein sollten. Aus der Arbeitsgruppe zur Erarbeitung der Festschrift im Rahmen des Jubiläumskomitees kam außerdem die Anregung, die Beiträge zweisprachig (deutsch und englisch) zu publizieren, um die Rezeption in einem internationalen Umfeld zu optimieren. Dem wurde gefolgt, was allerdings große Ansprüche sowohl an die Präzision der Darstellung als auch an die organisatorische Abwicklung stellte. Eine gesonderte Finanzierung der Forschungsarbeiten war nicht vorgesehen, doch wurden sowohl die Druckkosten als auch die administrative Unterstützung bei der Abwicklung durch das Jubiläumsbüro und die Finanzierung der Übersetzung der Texte ins Englische von der TU Wien übernommen.

Das Gesamtwerk umfasste schließlich 15 Einzelbände ${ }^{27}$, davon acht Bände, die den Fakultäten gewidmet waren und zwei Bände, die von der Universitätsbibliothek und der Hochschüler ${ }^{\star}$ innenschaft als weiteren wichtigen Stakeholdern gestaltet wurden. Zwei weitere Bände behandelten ergänzend kunst- und kulturgeschichtliche Themen: eine Geschichte der Rektorengalerie und Aspekte der Ausstrahlung der TU und ihrer Angehörigen in Kunst und Gesellschaft (Musik, Kunst, Fotografie, Kino, Erwachsenenbildung). Ein Band zur Baugeschichte war geplant, ließ sich aber nicht realisieren. Ein weiterer Band stellte die Organisationsgeschichte und Strukturentwicklung der TH/TU Wien der Jahre 1965 bis 2014 im Überblick dar, um so chronologisch den Anschluss an die 150-JahrFestschrift $\mathrm{zu}$ bieten. In einem umfangreichen, zweiteiligen Einleitungsband wurde schließlich von den wissenschaftlichen Mitarbeiter*innen des Archivs die Geschichte der TH Wien 1914-1955 behandelt, soweit sie sich aufgrund des derzeitigen Forschungsstandes darstellen lässt. Dieser Ausflug in die historische Ebene wurde vom Archiv vorgeschlagen und sollte bewusst als Ergänzung zu den beiden älteren großen Festschriften fungieren, die gerade diese Periode nicht einbeziehen konnten oder wollten. Damit fügte sich die 200-Jahr-Festschrift in das Gesamtkonzept der Jubiläumsfeierlichkeiten der TU Wien ein, das stark auf zukünftige Perspektiven und auf Kommunikation in die Gesellschaft hinein setzte. Der Umgang mit der eigenen Geschichte wurde von den Fakultäten sehr unterschiedlich gestaltet: Fallweise waren durchaus auch disziplingeschichtliche Ansätze vertreten, und insbesondere jene Fakultäten, die ihre Wurzeln bis in die

27 Seidler, Sabine (Hrsg.): Technik für Menschen. 200 Jahre Technische Universität Wien. 15 Bde. Wien 2015/2016. 
Gründungszeit des Polytechnischen Instituts zurückführen konnten, weiteten ihre historischen Betrachtungen auch darauf aus. Ganz offensichtlich bestand doch ein Bedarf an solchen „klassischen“ Rückblicken. ${ }^{28}$ Auch die im offiziellen Jubiläumskonzept bewusst weitgehend ausgesparte Hervorhebung ausgezeichneter Angehöriger des Hauses wurde in einem separaten Online-Projekt des AlumniVerbandes mit der wöchentlichen Vorstellung der Biographie eines bedeutenden Absolventen/einer Absolventin als „Hall of Fame“ doch noch realisiert. Hier war das Archiv ebenfalls unterstützend bei der Informationsbeschaffung und -Kontrolle eingebunden. ${ }^{29}$ Dagegen ließ sich eine historische Ausstellung, wie sie vom Archiv in früheren Jahren mehrfach und mit großer interner Resonanz durchgeführt worden war, 2015 aufgrund der räumlichen Situation - die TU Wien war wegen des „univercity“-Projekts faktisch eine Baustelle - und der knappen Personalressourcen nicht realisieren. Es ist auch fraglich, ob es dafür Unterstützung gegeben hätte.

Die „Jubiläumswebsite“ der TU Wien selbst hatte dagegen vor allem eine administrative Funktion: Sie steuerte die Kommunikation über das Programm und enthielt die Ankündigung zukünftiger und Berichte über vergangene Jubiläumsevents, historische Aspekte blieben dabei weitgehend ausgespart. ${ }^{30}$ Später wurde sie mit zusätzlichen Informationen zur TU Wien angereichert.

Das war an der Universität Wien ganz anders: Hier wurde - unter maßgeblicher Mitwirkung des Archivs der Universität Wien - eine umfassende Website zur Geschichte der Universität kreiert, die diese nach verschiedenen Themen, Personen, Aspekten und Zeiträumen an ein allgemeines Publikum vermitteln sollte. Die Geschichte der Institution erschien der Hochschule also durchaus als eine Ressource, die wissenschaftliche Qualität und ein entsprechendes Ansehen auch gegenüber der Öffentlichkeit beglaubigen kann. ${ }^{31}$ Dies signalisieren auch die Jubiläums-Slogans („Offen seit 1365“; „Offen für Neues seit 1365“; „Weitblick. Seit 1365“). Das Begleitprogramm des Jubiläumsjahrs verzeichnete ebenfalls eine ganze Reihe unterschiedlicher Ausstellungen und sonstiger Veranstaltungen mit

28 Besonders augenfällig wird dies beim Institut für Fertigungstechnik, das 2016, ein Jahr nach dem Jubiläum der TU Wien, sein eigenes 200-Jahr-Jubiläum veranstaltet, unter anderem mit einem großen Kongress, siehe Aigner, Florian: 200 Jahre Fertigungstechnik an der TU Wien. https://frei haus.tuwien.ac.at/200-jahre-fertigungstechnik-an-der-tu-wien/ (17.12. 2020).

29 Die Webseite wurde vom Alumni-Verband inzwischen fortgeschrieben und ist in der ursprünglichen Form nicht mehr abrufbar; vgl. TU Wien. Alumni Club: 100 Jahre Frauen an der TU Wien. https://www.tualumni.at/aktuelles/hall-of-fame/ (2.2.2021).

30 TU Wien: TU 200. 1815-2015. 200 Jahre Zukunft. http://www.tu200.at/ (2.2.2021).

31 Die, inzwischen überarbeitete und aktualisierte, Webseite 650 plus. Geschichte der Universität Wien. https://geschichte.univie.ac.at/de (17.12.2020). 
historischer Ausrichtung, die sich nicht nur nach „innen“, sondern auch nach „außen“, an ein allgemeines Publikum, wandten. ${ }^{32}$

Die umfangreiche Festschrift der Universität Wien ist dagegen klar an ein Fachpublikum gerichtet. Auch sie verfolgt nicht das Ziel einer „klassischen“ Festschrift, eine „traditionelle, vollständige oder repräsentative Institutionengeschichte“ zu liefern. Vielmehr setzte das vom „Forum Zeitgeschichte“ an der Universität Wien erarbeitete Konzept auf einen ambitionierten multiperspektivischen Ansatz, der von insgesamt mehr als 40 Fachhistoriker*innen umgesetzt wurde. Auch hier wurde der Betrachtungszeitraum eingegrenzt, und zwar auf die Zeit von der Mitte des 19. Jahrhunderts, als die Thun'schen Universitätsreformen begannen, bis zum Ende des 20. Jahrhunderts. Durch Anwendung von „forschungsorientierten und fächerübergreifenden Zugängen“ sollte der Versuch einer Neuausrichtung der Universitätsgeschichte zu einer „erweiterten Kulturgeschichte des Wissens“ unternommen werden. ${ }^{33}$ Die vier Bände sind jeweils großen Themenkomplexen gewidmet, von der Herausbildung einer neuen Wissensgesellschaft bis $\mathrm{zu}$ „reflexiven Innensichten“ (worunter sich dann doch eine Art „Fakultätenband“ mit einer biografischen „Porträtgalerie“ verbirgt - traditionelle Darstellungskonventionen wirkten also auch im Rahmen eines „reformorientierten“ Konzepts nach).

Dagegen legte die - sehr viel kleinere - Universität für Veterinärmedizin Wien eine einbändige, inhaltlich eher konventionell gestaltete Festschrift vor, die sich neben historischen Rückblicken, die auch die NS-Zeit explizit thematisieren, bewusst gegenwartsbezogenen Fragestellungen etwa zur Bedeutung des Berufsstandes der Tiermediziner und ethischen Problemen widmet. ${ }^{34}$ Das Jubiläum wurde hier außerdem zum Anlass genommen, schon ab 2014 ein dreijähriges Forschungsprojekt zur Geschichte der damaligen Tierärztlichen Hochschule in der Zeit des Nationalsozialismus zu beginnen. ${ }^{35}$ Die „neuen Medien“ wurden insofern genutzt, als eine „analog“ veranstaltete Ausstellung zur Geschichte der

32 Vgl. dazu Ash, Universitätsgeschichtsschreibung (wie Anm. 24), S. 230 - 233.

33 Stadler, Friedrich (Hrsg.): 650 Jahre Universität Wien. Aufbruch ins neue Jahrhundert. 4 Bde. Göttingen 2015, Zitate Bd. 1, S. 19 und 298; vgl. auch Ash, Universitätsgeschichtsschreibung (wie Anm. 24).

34 Haarmann, Daniela (Hrsg.): 250 Jahre Veterinärmedizinische Universität Wien. 1765-2015: 250 Jahre Verantwortung für Tier und Mensch. Wien 2015; online-Ausstellung und Jubiläumswebseite, die auch einen Rückblick auf weitere Veranstaltungen des Jubiläumsjahrs bietet: 250 Jahre Veterinärmedizinische Universität Wien. https://www.vetmeduni.ac.at/de/universitaet/ geschichte/250/ (17.12.2020).

35 Veterinärmedizinische Universität Wien: Aufarbeitung der NS-Zeit - FWF-Projekt. Die Tierärztliche Hochschule Wien im Nationalsozialismus. https://www.vetmeduni.ac.at/de/universi taet/geschichte/aufarbeitung/ (2.2.2021). 
Universität für Veterinärmedizin parallel als Internetpräsentation gezeigt wurde. Außerdem wurde ein Informationsvideo gedreht. Auch in diesem Falle fungierte die Geschichte als eine Legitimationsbasis für die heutige Bedeutungszuschreibung der Universität.

\section{Geschichte in Jubiläumszeiten - zwischen Werbe-Gag und institutioneller Selbstreflexion}

Im Vergleich der Jubiläumsgestaltung der TU Wien und der 2015 ebenfalls jubilierenden Wiener Universitäten lassen sich hinsichtlich der Inszenierungen und Mediennutzungen einige Gemeinsamkeiten feststellen. Allen Jubiläumskonzepten ist anzumerken, dass die jeweilige öffentliche Selbstpräsentation auch unter dem Diktat der Ressourcenkonkurrenz stand, insbesondere hinsichtlich der Anwerbung von Förderungen und der Weckung von nationaler wie internationaler Aufmerksamkeit. Hier schlägt sich die nachhaltige Ökonomisierung der Universitäten spätestens seit den Reformen ab 2004 nieder. Mit Markus Drüding ist festzuhalten, dass es beim Faktor „Ökonomie“ nicht nur um die Deckung der Kosten für den repräsentativen Konsum bei den Jubiläumsfeierlichkeiten geht (dieser Aspekt scheint bei früheren Jubiläumsfeiern stärker im Vordergrund gestanden zu haben), sondern mindestens genau so sehr darum, anlässlich des Jubiläums zusätzliche Mittel für den Ausbau der Universität einzuwerben. Feste kosten nicht nur Geld, sie können auch Geschenke einbringen. ${ }^{36}$

Alle drei Universitäten bedienten sich nicht nur der konventionellen analogen (Print-) Medien, sondern nutzten auf verschiedene Weise zusätzlich elektronische Kanäle, Social Media ebenso wie eigens eingerichtete Webseiten. Die eigene Geschichte war dabei in unterschiedlicher Form und Intensität präsent. Die jeweiligen Universitätsarchive waren dabei zumindest als „Inhaltslieferanten“ eingebunden, ihre Einwirkungsmöglichkeiten auf Inhalt und Gestaltung erscheinen jedoch begrenzt.

Aber es gab auch unterschiedliche Akzentsetzungen, insbesondere, was die Nutzung der eigenen Geschichte als Ressource betrifft. Die radikalste ,antihistorische“ Position wurde dabei von der TU Wien bezogen, die das Jubiläum

36 Vgl. Drüding, Markus: Warum feiern Universitäten Geschichte? Funktionen und Formen deutscher Universitätsjubiläen im späten 19. und 20. Jahrhundert. In: Akademische Festkulturen vom Mittelalter bis zur Gegenwart. Hrsg. von Martin Kintzinger, Wolfgang Eric Wagner u. Marian Füssel. Basel 2019 (Veröffentlichungen der Gesellschaft für Universitäts- und Wissenschaftsgeschichte 15). S. 55-76, hier S. 63-65. 
weitgehend unter das Thema „Zukunftsfähigkeit“ stellte. Hier hatte sich die Universitätsleitung also, um die Kategorisierung von Mitchell Ash aufzunehmen, für öffentliche Darstellung und politische Positionierung und nicht für die institutionelle „Selbstreflexion“ als Ziel des Jubiläums entschieden. ${ }^{37}$ Die eigene Geschichte und das Nachdenken darüber sollte bewusst keine herausragende Rolle spielen. Dass sich dies doch nicht immer ganz vermeiden ließ, hat mit dem genuin historischen Anlass der Festlichkeiten zu tun. Außerdem gaben sich aus dem Kreis der Universitätsangehörigen, etwa auf Fakultäts- und Institutsebene, auch Bedürfnisse nach „konventionelleren“ historischen Erzählungen zu erkennen, die zum Teil in den jeweiligen Beiträgen zur Festschrift realisiert wurden. Das Archiv hat in dieser Situation versucht, zumindest der jüngeren Geschichte des Hauses eine gewisse Präsenz zu sichern - wohl wissend, dass es dabei angesichts der weiterhin bestehenden Forschungslücken nur um eine vorläufige Bilanz gehen konnte.

In einer ähnlichen Position, was fachwissenschaftliche Vertretung und Personalausstattung des Archivs betrifft, befand sich die Universität für Veterinärmedizin, die sich jedoch für den konventionelleren Weg einer Festschrift unter Einbeziehung ihrer geschichtlichen Entwicklung von der Gründung bis zur Gegenwart entschied. Eine Ausstellung mit Online-Präsentation sollte wohl die Reichweite der Selbstpräsentation erweitern. Außerdem nützte sie die „Jubiläums-Konjunktur“ für die Beantragung eines Forschungsprojekts zur NS-Geschichte. Das Archiv fungierte hier in erster Linie als „Content-Lieferant“.

Dagegen versuchte sich die Universität Wien, ausgestattet mit umfangreicher fachhistorischer Expertise, in der Festschrift an einem methodologischen Neuzugang zur Universitätsgeschichtsschreibung, wobei die zeitliche Limitierung auf die Zeit seit der Mitte des 19. Jahrhunderts nicht unbedingt frei gewählt, sondern auf Wunsch des Rektorats erfolgt war. Darüber hinaus brachten Universitätsarchiv und Fachwissenschaftler ${ }^{\star}$ innen ihre historische Kompetenz auch bei zahlreichen weiteren Events und Präsentationsformen ein.

Gemeinsam ist allen drei Universitäten auch die Ausdehnung der JubiläumsFeiern auf das ganze Jahr 2015. Ein langer Reigen verschiedenster Veranstaltungen und Events, sei es eigens für den Anlass entwickelt oder nur für das Jubiläum „gebrandet“, zog sich durch das Jahr, wobei die Feier des jeweiligen Gründungstages mit dem eigentlichen akademischen Festakt als Höhepunkt fast ein wenig unterging. Eine festliche Differenz zum „Alltag“38 war bei dieser zeitlichen Erstreckung kaum aufrecht zu erhalten. Diese machte es aber auch notwendig, die

37 Ash, Universitätsgeschichtsschreibung (wie Anm. 24), S. 222 f.

38 Vgl. Drüding, Universitäten (wie Anm. 36), S. 58. 
Organisation nicht mehr, wie es früher üblich gewesen war, allein ehrenamtlich von Universitätsangehörigen durchführen zu lassen, sondern sie einem professionellen Eventmanagement anzuvertrauen.

Typologisch zeigt sich bei den Wiener Jubiläumsfeiern des Jahres $2015 \mathrm{im}$ Vergleich zu 1965 ein markanter Bruch im Auftreten der Universitäten gegenüber der Öffentlichkeit: Auch in Österreich wurde damals der „politische Systemwechsel“ - und der gelungene Wiederaufbau - „mit der Konstruktion einer neuen historischen Tradition beantwortet“. ${ }^{39}$ Dabei griff man stark auf ältere Festgebräuche zurück - sinnfällig etwa daran, dass sich sowohl die Universität Wien als auch die damalige TH Wien für diesen Anlass neue Talare entwerfen und anfertigen ließen. Dagegen können die Feiern von 2015 dem von Sylvia Paletschek georteten „neuen Konsens“ zugeordnet werden, der, nach einer Phase der Zurückhaltung in den 1970er und 1980er Jahren, wieder aufwändige Feierlichkeiten zuließ, diese aber nun deutlich bewusster als Instrumente der Eigen-PR nach außen und der Stärkung der „Corporate Identity“ nach innen einsetzte. ${ }^{40}$ Auch die verstärkte Aufarbeitung der eigenen NS-Vergangenheit kann unter diesem Aspekt gesehen werden, manches Projekt wäre ohne die „Jubiläums-Perspektive“ so vielleicht nicht möglich gewesen.

39 So in Bezug auf die Situation im geteilten Deutschland Paletschek, Sylvia: Festkultur und Selbstinszenierung deutscher Universitäten. In: Mittendrin - Eine Universität macht Geschichte. Eine Ausstellung anlässlich des 200-jährigen Jubiläums der Humboldt-Universität zu Berlin. Hrsg. von Ilka Thom, Kirsten Weining u. Heinz-Elmar Tenorth. Berlin 2010. S. 88-95, hier S. 93; die Beobachtung lässt sich auch auf Österreich übertragen.

40 Paletschek, Festkultur (wie Anm. 39), S. 95. 
